\title{
Crecimiento económico y el uso de energía sustentable y no sustentable: un enfoque del caso ecuatoriano usando técnicas de cointegración
}

\section{Economic growth and the use of sustainable and unsustainable energy: an Ecuadorian case approach using cointegration techniques}

\author{
Verónica Stefanía Loaiza Godoy ${ }^{1}$ \\ ${ }^{1}$ Universidad Nacional de Loja \\ *verito_04teffy@hotmail.com
}

DOI: https://doi.org/10.26871/killkana_social.v2i3.326

\begin{abstract}
Resumen
La amenaza del cambio climático y la dependencia de las importaciones energéticas, junto al carácter agotable de los combustibles fósiles, han incentivado a muchos países a buscar fuentes de energía alternativas, esto con el fin de reducir las emisiones de gases efecto invernadero. Por ello, esta investigación analiza la relación de equilibrio existente entre la energía sustentable, no sustentable y el crecimiento económico de Ecuador durante el periodo 1971-2014. Utilizando datos del World Development Indicators del Banco Mundial y técnicas de cointegración para series temporales, encontramos evidencia que muestra que existe una relación de largo y corto plazo entre la energía sustentable, energía no sustentable y el crecimiento económico. También encontramos que existe una relación causal unidireccional desde la energía sustentable hacia el crecimiento económico. Lo que nos lleva a concluir que esta variable es de suma importancia para que exista mayor crecimiento económico, por esta razón hoy en día muchos países desarrollados están invirtiendo cada vez más en este tipo de energías limpias. Además de ayudar a disminuir la contaminación, también su costo está muy por debajo de las energías convencionales. Para el caso de Ecuador, ya se está trabajando en el cambio de la matriz energética, evidenciando grandes obras como los ocho proyectos hidroeléctricos, la mayoría de ellos ya en funcionamiento, por esta razón Ecuador se ha convertido en un país exportador de energía limpia. Una implicación política derivada de esta investigación es fortalecer tanto la oferta como la demanda de energías sustentables como mecanismo para robustecer el crecimiento económico de países que se encuentran en desarrollo.
\end{abstract}

Palabras clave: Crecimiento económico, Energía renovable, no renovable, Ecuador.

\begin{abstract}
The threat of climate change and the dependence on energy imports, together with the exhaustible nature of fossil fuels, have encouraged many countries to look for alternative sources of energy, in order to reduce greenhouse gas emissions. Therefore, this research analyzes the equilibrium relationship between sustainable, unsustainable energy and Ecuador's economic growth during the period 1971-2014. Using data from the World Bank's World Development Indicators and cointegration techniques for time series, we find evidence showing that there is a long and short term relationship between sustainable energy, non-sustainable energy and economic growth. We also find that there is a unidirectional causal relationship from sustainable energy to economic growth. Which leads us to conclude that this variable is of the utmost importance for there to be greater economic growth, for this reason today many developed countries are investing more and more in this type of clean energy. In addition to helping to reduce pollution, its cost is also far below conventional energies. In the case of Ecuador, work is already underway to change the energy matrix, evidencing major works such as the eight hydroelectric projects, most of them already in operation, for this reason Ecuador has become a clean energy exporting country. A political implication derived from this research is to strengthen both supply and demand of sustainable energies as a mechanism to strengthen the economic growth of countries that are developing.
\end{abstract}

Key words: Economic growth, Renewable energy, non-renewable, Ecuador. 


\section{Introducción}

Según datos World Development Indicators (WDI, 2017) del Banco Mundial, el uso promedio de energía no renovable per cápita aumentó desde 1971 siendo el valor promedio en este año de $1336.709 \mathrm{~kg}$ de equivalente de petróleo per cápita y pasando a $1920.724 \mathrm{~kg}$ de equivalente de petróleo per cápita en 2014. Estas cifras repercuten sobre las emisiones de $\mathrm{CO}_{2}$ globales, ya que según datos de la Agencia Internacional de la Energía (IEA, 2015) en su informe denominado World Energy Outlook, las emisiones de $\mathrm{CO}_{2}$ a nivel mundial se mantuvieron tres años seguidos en 32.100 millones de toneladas hasta el año 2015 aunque estas cifras generan expectativas positivas por la ralentización de estos indicadores, las proyecciones sobre emisiones de $\mathrm{CO}_{2}$ resultante de la combustión de los derivados fósiles en todo el mundo son devastadoras si no se pone énfasis y seguir reduciéndolas, por ejemplo, las emisiones de $\mathrm{CO}_{2}$ por parte de los países de la de la Organización para la Cooperación y el desarrollo Económico (OCDE) contribuirán alrededor de 13.800 millones de toneladas métricas en 2040 (AIE, 2015).Estas cifras reveladoras y preocupantes, evocan la necesidad de un modelo económico bien equilibrado que ayude a generar modelos de crecimiento económico sustentable con el ambiente. Según la IEA (2015), la electricidad generada por las renovables ha desempeñado un papel importante, al alcanzar el $90 \%$ de la generación de nueva electricidad en 2015 a nivel mundial, en donde la energía eólica fue la responsable de más de la mitad de esa nueva electricidad generada.

Desde luego dentro de lo que se refiere a energías renovables, las centrales hidráulicas dominan el sector, con $1.096 \mathrm{GW}$, pero el resto de segmentos aumentan a buen ritmo. La eólica es la siguiente, con $487 \mathrm{GW}$, seguida de la solar, que cuenta ya con 303 GW de capacidad. El Renewable Energy Policy Network (REN 21), es la red mundial de políticas en energía renovable que conecta a un gran número de actores clave. La meta de REN 21 es facilitar el intercambio de conocimiento, el desarrollo de políticas y la suma de esfuerzos para una transición mundial rápida hacia la energía renovable, un informe realizado por esta red revela que la inversión en fuentes de energías renovables a nivel mundial, alcanzo los 285.900 millones de dólares (REN21, 2015) que representa el doble que se destina a combustibles fósiles, observando así el crecimiento acelerado de energía limpia.

China, el mayor inversor a nivel mundial de energía renovable, representa el $37 \%$ del total mundial, esto es el resultado de políticas públicas que ayuden a promover generación de nuevas fuentes de energía alternativas, dado que este país a partir del 2005 cuenta con una ley de promoción de energía renovable. Aunque el gobierno chino se ha empeñado en fortalecer el uso de energías sustentables, existen varios estudios realizados en torno a este país, donde existen altas tasas de contaminación, específicamente en las regiones orientales, occidentales y centrales por el uso de energía no sustentables específicamente el carbón.
El desarrollo de las energías renovables y la eficiencia energética supone üna nueva era de exploración energética. ${ }^{\text {en }}$ los Estados Unidos, así lo expuso el ex Presidente Barack Obama, ya que este país fue el mayor inversor hasta el 2009, pero la falta de políticas estatales que fomenten la producción de energía lo hizo descender a segundo lugar. La capacidad instalada en EE.UU. de energía eólica supera ya los 43.460 MW y suministra el $3 \%$ de la electricidad del país (REN21, 2015). Texas, está firmemente establecida como el líder en el desarrollo de energía eólica, seguido por Iowa y California, además EE. UU fue pionero en tecnología con el proyecto One.5 solar, y se han construido varias estaciones de energía solar térmica. La mayor de estas centrales térmicas solares es el Grupo de plantas SEGS6 en el desierto de Mojave, con una capacidad total de generación de $354 \mathrm{MW}$, por lo que se ha convertido el sistema en la mayor planta solar de cualquier tipo en el mundo.

En América Latina si excluimos a Brasil, que es el líder indiscutible, hace 10 años la inversión regional en energía renovable era de US\$1.000 millones al año. Mientras que en los últimos tres años ha aumentado a US\$6.000, US\$7.1000 y US\$9.3000 millones respectivamente, después de Brasil tenemos países como México, Chile y Uruguay, así que definitivamente hay una curva de crecimiento en Latinoamérica. En este sentido, Ecuador también ha logrado posicionarse como un referente mundial, figurando como quinto en seguridad energética según un informe de la Universidad de Vancouver, y sobre todo se destaca por sus inmensos esfuerzos en materia de construcción de sus ocho proyectos hidroeléctricos. Ecuador gracias al cambio de la matriz energética y a la importante inversión que realizo el gobierno, se ha logrado remplazar el consumo de combustibles fósiles por la producción de energía renovable que actualmente representa el $51,78 \%$. El propósito del gobierno es alcanzar el $93 \%$ de energía limpia y renovable, aprovechando el potencial de los recursos naturales como los hídricos, solares, eólicos; y de esta manera ir desechando gradualmente la producción de energía contaminante. La principal obra denominada Coca Codos, permitirá al país ser un potencial exportador de energía en la región, rezagando así la dependencia energética que tenía el país con Colombia y Perú.

En la presente investigación nos hemos enfocado en analizar la relación que existe entre las energías renovables, energías no renovables y el crecimiento económico, con la finalidad de comprobar la hipótesis de que el crecimiento económico de Ecuador es dependiente de la energía no sustentable, lo que a su vez implica una mayor contaminación ambiental. Existe una amplia literatura que realiza este tipo de relación en diferentes países del mundo. Los autores Taghvaee, Mavuka, y Shirazi (2016) realizan una relación entre el crecimiento económico y los diferentes tipos de energías, donde encontraron que la relación entre el crecimiento económico y el consumo de energía en Irán, considerando los diversos tipos de energía ya mencionados, 
es negativa, estos resultados explican la ineficacia de las políticas de deflación cuantitativas y cualitativas sobre el sector de la energía, es decir, que ni el decreciente consumo de energía ni el cambio en la cartera de energía, afectan el crecimiento económico de este país, los autores sugieren que se debería formular políticas medioambientales que reduzcan la cantidad de consumo de energía no renovable, ya que no tienen consecuencias negativas considerables en el crecimiento económico, mientras que aumentar las energías renovables en la cartera de consumo de energía, mejorara tanto la calidad ambiental como la seguridad energética. En esta nuestra investigación los resultados arrojan evidencia de que existe una relación de corto y largo plazo entre las variables del modelo planteado, además existe una relación causal unidireccional desde la energía sustentable hacia el crecimiento económico.

Esta investigación contribuye con evidencia empírica robusta sobre la relación entre consumo de energía sustentable y no sustentable con el crecimiento económico en el Ecuador, un país en vías de desarrollo que tiene una baja participación manufacturera, lo cual facilita el uso de energía proveniente de fuentes sustentables. La estimación de un modelo de cointegración y de corrección de error lleva a obtener resultados consistentes de la relación de corto y largo plazo entre las variables. Asimismo, este documento emplea el método de Causalidad de Granger (1969) con el fin de determinar si existe causalidad entre las variables. Efectivamente, demostramos que existe causalidad unidireccional, es decir la energía sustentable causa el crecimiento económico. Y lleva a concluir que el Ecuador tiene un potencial muy alto para explotar energía sustentable a través del cambio de la matriz energética. En general, estos resultados sugieren que el uso de energía procedente de fuentes sustentables no limita el crecimiento económico de Ecuador.

La presente investigación está estructurada en cuatro secciones adicionales a la introducción. En la segunda sección contiene una breve revisión de la literatura previa. En la tercera describimos los datos y la metodología utilizada, en donde derivamos el modelo teórico. En la cuarta discutimos los resultados encontrados. En la quinta sección constan las conclusiones de la investigación y las posibles implicaciones de política económica.

\section{Revisión de literatura}

Tomando en cuenta que no existe una teoría que englobe una relación entre las variables propuestas en esta investigación. La evidencia empírica en este campo se ha extendido en los últimos años dado que se ha vuelto imprescindible cambiar el actual modelo energético para hacerlo sostenible y esto implica erradicar las fuentes de energía más contaminantes y peligrosas para el medio ambiente en que vivimos. En base a esto Salim, Hassan y Sha (2014) en su investigación propuesta utilizando pruebas de raíz unitaria, cointegración y causalidad de Granger. Los resultados arrojaron una relación positiva a largo plazo entre las fuentes de energía renovable y no renovable, la producción industrial y el crecimiento económico. Existe causalidad unidireccional entre el crecimiento del PIB y el consumo de energía renovable. Estos resultados indican que los países de la OCDE, aún siguen siendo dependientes de la energía principalmente para su producción industrial. En este sentido la expansión de las fuentes de energía renovable es una solución viable para abordar la seguridad energética y sobrellevar los problemas del cambio climático, y la sustitución gradual de fuentes de energía renovables por fuentes de energía no renovable, esto podría mejorar el crecimiento económico de un país.

En la mayoría de los principales países exportadores de petróleo, las políticas gubernamentales mantienen los precios internos por debajo del nivel del mercado libre, lo que da lugar a altos niveles de consumo de energía nacional. Mehrara (2007) propone una relación positiva entre el consumo de energía y crecimiento económico, tomando el caso de los países exportadores de petróleo. Los resultados implican que la conservación de la energía mediante la reforma de las políticas de precios de la energía no tiene repercusiones perjudiciales sobre el crecimiento económico de este grupo de países.

Con fines comparativos dividiremos la evidencia empírica en tres grupos. Tomando en cuenta que los países más desarrollados consumen mayor cantidad de energía que los países menos desarrollados, y por ende registran mayores tasas de contaminación. Bajo esta premisa, se ha tomado en cuenta en el primer grupo a los países de ingresos altos, el segundo grupo se incluyen países de ingresos bajos y finalmente agruparemos las investigaciones donde estiman la misma relación, para diferentes grupos de países.

Dentro del primer grupo, una de las grandes potencias económicas del mundo y por ende un país con ingresos altos como lo es China, se evidencia altas tasas de contaminación, específicamente en las regiones orientales, occidentales y centrales, derivadas del uso de energías no sustentables, específicamente el carbón, Kang, Zhao y Yang (2016) establecen en su investigación, que el crecimiento económico si está relacionado con el consumo de energía no sustentable a largo plazo, pero además de aumentar las emisiones de $\mathrm{CO}_{2}$, también perjudican el crecimiento de la economía. Por esta razón el autor sugiere que el gobierno debe implementar políticas para reducir las emisiones de $\mathrm{CO}_{2}$, utilizando las nuevas innovaciones tecnológicas para crear energía sustentable. Tras varias décadas de crecimiento económico, China se ha convertido en el mayor consumidor de energía y el mayor emisor de $\mathrm{CO}_{2}$ en el mundo. Bajo esta premisa Wang et al. (2016) establecieron en su investigación que es necesario comprender mejor la relación entre el crecimiento económico, el consumo de energía y las emisiones de $\mathrm{CO}_{2}$.

Es clave desarrollar energía hidroeléctrica y nuclear en China, así lo establecen Long, Yaw, Du y Zhuang (2015), tras realizar un análisis de cointegración, pruebas de raíz unitaria y un análisis de causalidad de Granger. 
Chiou et al. (2012) encuentran evidencia que apoya una hipótesis de neutralidad para los Estados Unidos, Tailandia y Corea del Sur. Sin embargo, las pruebas empíricas sobre Filipinas y Singapur revelan una causalidad unidireccional que va desde el crecimiento económico hasta el consumo de energía, mientras que el consumo de energía puede haber afectado el crecimiento económico de Taiwán, Hong Kong, Malasia e Indonesia.

Siguiendo con el primer grupo, Estados Unidos un país rico, poderoso e influyente del mundo, pero con un alto grado de contaminación, se encuentra ubicado en el puesto número dos del Rankin de países con mayores emisiones de $\mathrm{CO}_{2}$, siendo el primero China. Tomando en cuenta estos antecedentes Bowden y Payne (2009) examinaron la relación causal entre la energía y el PIB real, donde encontraron que causalidad bidireccional está presente entre la energía primaria comercial y residencial con el PIB real. Los resultados sugieren que las políticas energéticas y ambientales prudentes deberían reconocer las diferencias en la relación entre la energía y el PIB real. Con una dinámica económica parecida a la del país anterior, Canadá cuenta con una industria de alta tecnología además de una economía sólida y con un PIB superior al billón de dólares. Ghali (2004) utilizando un modelo VEC, encontró que la dinámica de corto plazo de las variables indica que la causalidad bidireccional entre el crecimiento de la producción y el uso de energía, con estos resultados una implicación política importante es que la energía puede considerarse como un factor limitante para el crecimiento del PIB en Canadá.

Irán, al igual que Ecuador, es un país con una economía mixta y altamente dependiente de las exportaciones de hidrocarburos. Los autores Taghvaee, Mavuka y Shirazi (2016) establecen una relación entre el crecimiento económico con los diversos tipos de energías, estimando un modelo autorregresivo de rezagos distribuidos (ARDL). Encontraron que la relación entre el crecimiento económico y el consumo de energía en Irán, considerando los diversos tipos de energía, es negativa, estos resultados sugieren que ni el decreciente consumo de energía ni el cambio en la cartera de energía afectan el crecimiento económico. Concluyendo que aumentar las energías renovables en la cartera de consumo de energía, mejorara tanto la calidad ambiental como la seguridad energética pero no afectara al crecimiento económico.

En Australia, Salahuddin y Alam (2015) establecen una relación entre los efectos a corto y largo plazo del uso de Internet y el crecimiento económico en el consumo de electricidad. Aplican la prueba ARDL de límites para la cointegración. Los resultados de las estimaciones del modelo ARDL indican que el uso de Internet y el crecimiento económico estimulan el consumo de electricidad. El uso de Internet y el crecimiento económico no tienen una relación significativa a corto plazo con el consumo de electricidad. La prueba de causalidad de Granger multivariante confirma el vínculo causal unidireccional que va desde el uso de Internet hasta el crecimiento económico y el consumo de electricidad, además también se recomienda una coordinación entre las políticas de Tecnologías de la información y la comunicación (TIC), la política energética y la política de crecimiento.

Grecia la ciudad más grande de Atenas, también ha sido objeto de este tipo de estudios Dergiades, Martinopoulos y Tsoul (2013) encuentran resultados empíricos que revelan conexiones causales unidireccionales, tanto lineales como no lineales, que van desde la energía útil total hasta el crecimiento económico, esos resultados permitirán que se puedan implementar políticas energéticas más efectivas con respecto a la protección energética y ambiental. Estos resultados coinciden con los resultados propuestos por Hondroyiannis, Lolos y Papapetrou (2002) quienes también analizan el caso de Grecia.

En el segundo grupo, comenzaremos hablando de los países de ingresos bajos que pertenecen al continente asiático en Malasia Aziz (2011) examina mediante el uso de los modelos de cointegración y corrección de error vectorial (VEC), donde los resultados muestran la existencia de una relación a largo plazo entre el consumo de energía, los precios de la energía y el crecimiento económico. Los resultados también muestran causalidad unidireccional que el crecimiento económico ejerce sobre la energía. La implicación política debe estar dirigida principalmente a la de conservación de energía, que puede implementarse sin poner en riesgo el desarrollo económico.

Vietnam es un país soberano del Sudeste Asiático donde los autores Foon, Wah y Ozturk (2016) analizan la relación entre energía y el crecimiento económico, con los métodos de cointegración y causalidad de Granger encuentran resultados que confirman la existencia de cointegración entre las variables, además de encontrar causalidad unidireccional que va desde la energía hasta el crecimiento económico. Es importante mencionar que Vietnam es una economía dependiente de la energía, por esta razón, la política de energía renovable se debe dar para proporcionar suficientes suministros de energía para acelerar la expansión económica.

Para el caso de Nepal, otro país que pertenece al continente asiático, Bastola y Sapkota (2015) examinan las relaciones causales entre la energía, la emisión de contaminantes y el crecimiento económico, empleando una metodología econométrica de series de tiempo. Encontraron causalidad unidireccional que va desde el crecimiento económico hasta las emisiones de carbono y el consumo de energía. Estos hallazgos recomiendan que las políticas que impulsan el consumo de energía no son crecimiento económico, sino que tienen más probabilidades de ejercer efectos adversos sobre el medio ambiente. Estos resultados son coincidentes con los resultados del caso Nigeria realizado por Maji (2015).

En el último grupo se integran todos los trabajos que incluyen varios países a la vez. Existen varios estudios que toman como referencia los países que pertenecen a la OCDE. Algunos autores han realizado sus investiga- 
ciones tomando en cuenta los datos de estos países. Por ejemplo Salim, Hassan y Sha (2014)) ; Belke, Dobnik y Dreger (2011), exploran los determinantes de las emisiones de $\mathrm{CO}_{2}$ utilizando un modelo STIRPAT , donde los resultados empíricos muestran que el consumo de energía renovable disminuye las emisiones de $\mathrm{CO}_{2}$. Además, los resultados respaldan la existencia de una curva ambiental de Kuznets entre la urbanización y las emisiones de $\mathrm{CO}_{2}$, lo que implica que a niveles más altos de urbanización, el impacto ambiental disminuye. Al-mulali, Fereidouni y Lee (2014) emplean el modelo vectorial de corrección de errores (VEC), donde se incluye el consumo de energía, el PIB real y la evolución de los precios, este último tomado para representar una medida de la eficiencia económica. Los resultados indican que existe una relación a largo plazo entre las tres variables, que respalda la endogeneidad de la energía y el producto real, este estudio sugiere que se adopten políticas estructurales adecuadas destinadas a mejorar la eficiencia económica, donde se podría inducir a la conservación de la energía sin impedir el crecimiento económico.

Algunos autores han realizado investigaciones con la misma relación, pero además utilizan la curva de Kuznets (Saboori y Sulaiman, 2013; Ben, Ben, y Ozturk, 2017; Pablo-Romero y De Jesús, 2016), estudios en donde se encuentra que el consumo de energía renovables conduce al aumento del crecimiento económico, además que un desarrollo tecnológico puede resultar en un favorable crecimiento económico. Finalmente se acepta la hipótesis de que efectivamente se cumple la curva de Kuznets en estos países. De la misma manera Ohler y Fetters (2014), Con un modelo de corrección de errores de panel, concluyeron que la energía renovable y la hidroelectricidad incrementan el PIB.

Salim et al., (Salim y cols., 2014) examinan la relación entre el consumo de energía renovable y no renovable, el producto industrial y el crecimiento del PIB en los países de la OCDE. Los resultados muestran que existe una relación de equilibrio a largo plazo entre las fuentes de energía no renovables y renovables, la producción industrial y el crecimiento económico. Encuentran también la existencia de causalidad bidireccional entre el producto industrial y las energías renovables y no renovables a corto y largo plazo. Zaman et al. (2016) encontraron en su investigación que las variables ambientales tienen un efecto perjudicial sobre el crecimiento económico de las economías de Brasil, Rusia, India, China y Sudáfrica (BRICS), mientras que las fuentes de energía aumentan significativamente el crecimiento económico. Una relación causal bidireccional entre el consumo de energía y el crecimiento económico ha sido identificada a largo y corto plazo para todos los países de la Unión por el mediterráneo, que reaccionarían de manera similar a las políticas energéticas (Esseghir y Haouaoui Khouni, 2014). En piases del pacifico Fang y Chang (2016) confirman que los resultados varían entre países y se muestra que el capital físico y energía contribuyen significativamente al desarrollo económico en los países asiáticos del pacifico. Bowden y Payne (2009) utilizando el modelo de corrección de error de panel, encuentran resultados que revelan causalidad bidireccional entre el consumo de energía renovable, no renovable y el crecimiento económico, tanto a corto como a largo plazo.

En términos generales, El PIB depende considerablemente de la energía no sustentable, lo que es alarmante, ya que este tipo de energía a más de contaminar el medio ambiente donde vivimos tiene un costo monetario y ambiental muy elevado. En el caso de Ecuador, no se han realizado estudios que permitan encontrar si existe o no una relación a largo y corto plazo entre la energía sustentable, no sustentable y el crecimiento económico.

\section{Datos y Metodología}

\subsection{Datos}

Esta investigación utiliza datos de series de tiempo anuales tomados del World Development Indicators (WDI, 2016) del Banco Mundial, periodo 1970-2014 para Ecuador. La variable dependiente es el PIB per cápita y se utiliza como una variable proxi del crecimiento económico, y las variables independientes son la tasa de consumo de energía eléctrica como proxy de Energía sustentable y la tasa del uso de energía derivada del petróleo utilizada como una variable proxy de Energía no sustentable, a continuación, la Tabla 1 describe las variables mencionadas.

Tabla 1 Descripción de las variables

\begin{tabular}{|c|c|c|c|}
\hline Variable & Nombre & Símbolo & Descripción \\
\hline Dependiente & $\begin{array}{l}\text { Crecimiento } \\
\text { del PIB per } \\
\text { cápita ( } \% \\
\text { anual) }\end{array}$ & PIBpc & $\begin{array}{l}\text { Relación entre el valor total } \\
\text { de todos los bienes y servicios } \\
\text { finales generados durante un } \\
\text { año por la economía de una } \\
\text { nación o estado y el número de } \\
\text { sus habitantes en ese año. }\end{array}$ \\
\hline \multirow[t]{2}{*}{ Independientes } & $\begin{array}{l}\text { Consumo de } \\
\text { energía } \\
\text { eléctrica \% } \\
\text { (kW per } \\
\text { cápita) }\end{array}$ & ES & $\begin{array}{l}\text { Taza de consumo de energía } \\
\text { eléctrica, medida en } \mathrm{kW} \text { per } \\
\text { cápita }\end{array}$ \\
\hline & $\begin{array}{c}\text { Uso de } \\
\text { energía \% }(\mathrm{kg} \\
\text { de equivalente } \\
\text { de petróleo per } \\
\text { cápita) }\end{array}$ & ENS & $\begin{array}{l}\text { Taza de consumo de energía } \\
\text { derivada del petróleo, medida } \\
\text { en Kg equivalente de petróleo } \\
\text { o per cápita }\end{array}$ \\
\hline
\end{tabular}

Previo al análisis econométrico de los modelos de vectores auto regresivos (VAR) y modelos de corrección de error (VEC), este último en caso que exista un equilibrio a corto plazo, se elaboró un análisis descriptivo y de correlación de las variables. La Figura 1 muestra el comportamiento de las tres variables incluidas en el modelo econométrico, las variables tienen un comportamiento cíclico, lo que nos lleva a pensar que las series son estacionarias. Para verificar lo antes descrito aplicaremos el test de Dickey y Fuller (1979). 
Figura 1. Evolución de las variables en niveles en el periodo 1970-2014.
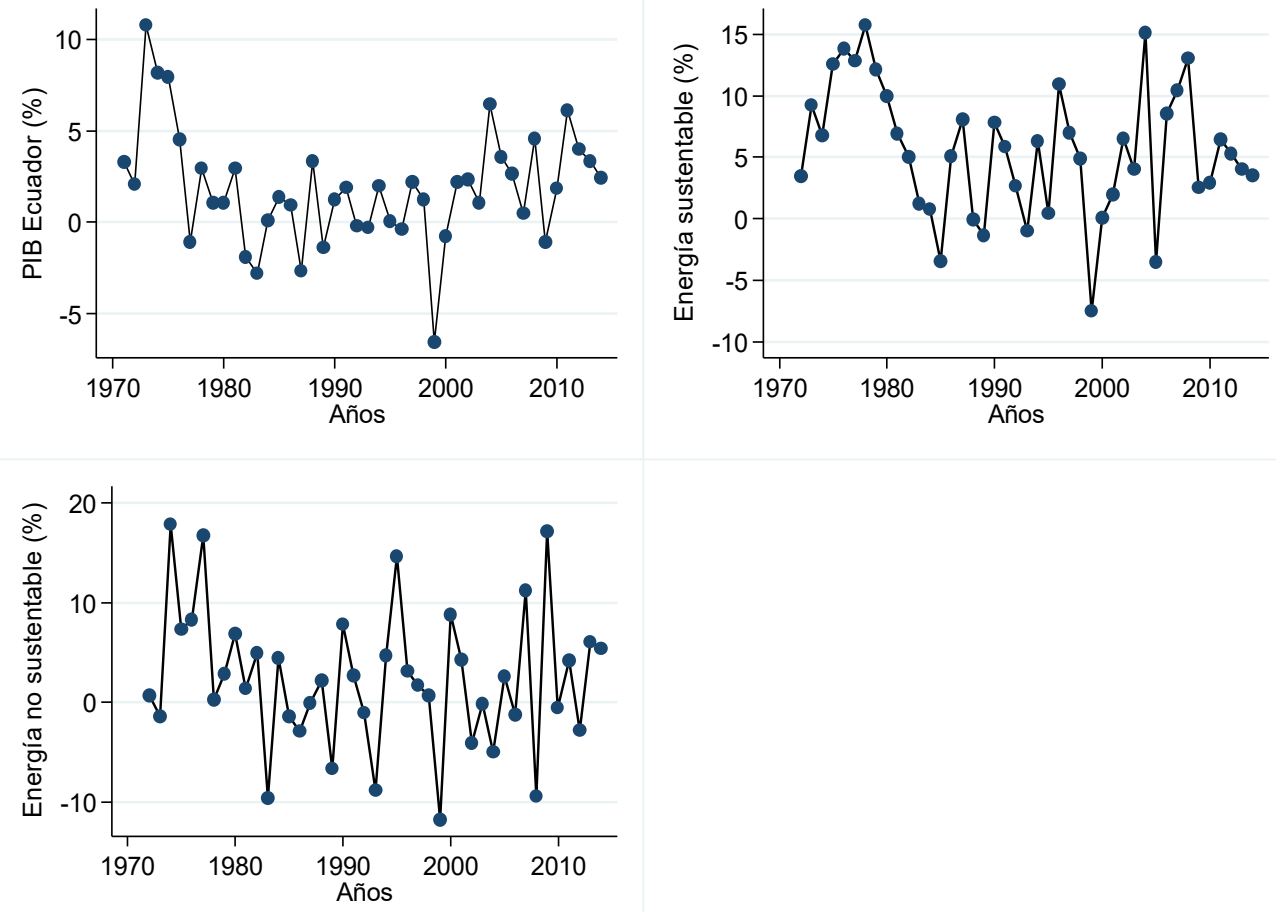

Fuente: Elaboración propia con datos Banco Mundial (2014).

La Figura 2 muestra la correlación entre las variables del modelo econométrico, podemos observar que existe una correlación negativa entre el PIB per cápita y Energía sustentable, y de igual manera una correlación negativa entre el PIB per cápita y Energía no sustentable

Figura 2. Correlación entre las variables del modelo
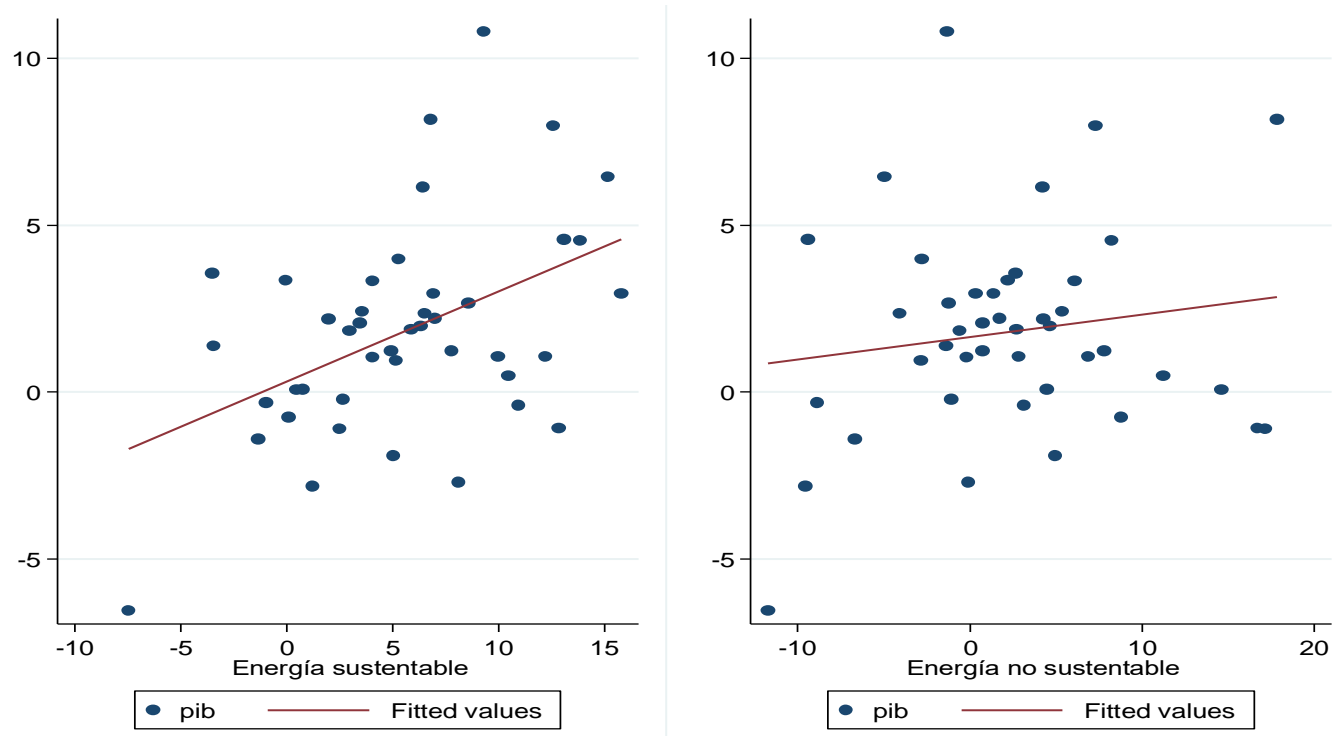

Fuente: Elaboración propia con datos Banco Mundial (2014). 
En la Figura 3 muestra el gráfico de impulso -respuesta de las variables crecimiento económico, energía sustentable y energía no sustentable para el caso de Ecuador. En la figura del lado izquierdo observamos que el shock del crecimiento económico frente a la respuesta de la energía sustentable disminuye simultáneamente hasta diluirse. En la figura de la derecha se observa un pequeño incremento en el primer año del crecimiento económico frente a un shock fuerte en la energía no sustentable, para el segundo año tiene una caída importante y a partir de este año el efecto comienza a disminuir y al final se diluye. Esto es en consecuencia de la fuerte inversión en el cambio de la matriz energética. Aumentando el crecimiento económico del país ya que además de consumir energía limpia y más barata, también el país exportará a otros países, lo que dará paso a ingreso de divisas al país por otro rubro distinto a la exportación de petróleo.

Figura 3. Resultado del test de Impulso- Respuesta
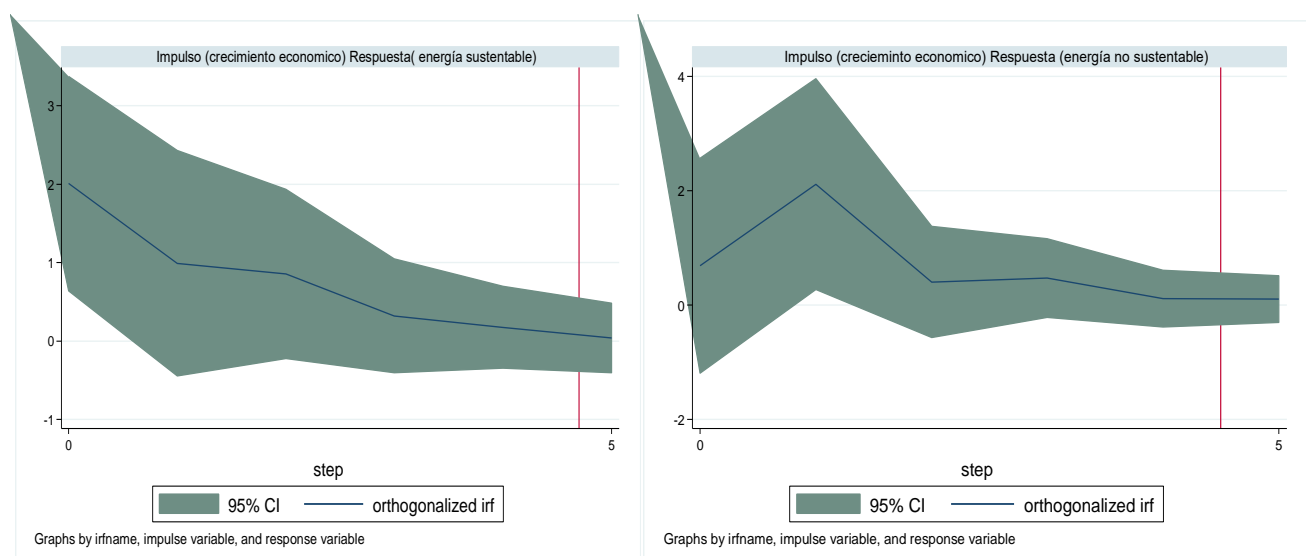

Fuente: Elaboración propia con datos Banco Mundial (2014).

\subsection{Metodología}

Con el fin de verificar econométricamente la relación existente entre el crecimiento económico (PIBpc), la tasa de consumo de energía eléctrica (ES) y la tasa de consumo de energía derivada del petróleo (ENS) para Ecuador tenemos la siguiente función:

$$
P I B p c_{t}=f\left(E S_{t}, E N S_{t}\right)
$$

Donde Producto Interno Bruto per cápita $P I B p c_{t}$ del país i $(\mathrm{i}=1,2, \ldots, \mathrm{I})$ en el período $\mathrm{t}(\mathrm{t}=1970,1971$, . . . , T) está en función de la tasa de consumo de energía eléctrica $E S_{t}$ y la tasa de consumo de energía derivada del petróleo $E N S_{t}$ y describe una relación causal hacia el PIB per cápita. A partir de la ecuación (1) se deriva el modelo MCO:

$$
Y_{t}=\beta_{0}+\beta_{1} X_{1 t}+\beta_{2} X_{2 t}+\varepsilon_{t}
$$

Para el caso de Ecuador es necesario incluir una variable dummy que capture el cambio estructural que sufrió el Ecuador en los años 1999-2000 al adoptar el dólar como moneda oficial, hemos planteado la siguiente ecuación:

$$
P I B p c_{t}=B_{0}+B_{1} E S_{t}+B_{2} E N S_{t}+B_{3} D u m m y_{t}+u_{t}
$$

Donde $P I B p c_{t}$ representa la tasa de crecimiento del Producto interno bruto per cápita, $B_{1}$ mide el efecto de consumo de energía sustentable (ES) en el crecimiento económico, $B_{2}$ mide el efecto de la tasa de consumo de energía no sustentable $\left(E N S_{t}\right)$ en el crecimiento económico y $B_{3}$ mide el efecto de la variable dummy, que representa el cambio estructural experimentado por la crisis económica y financiera entre los años 1999-2000, y finalmente $u_{t}$ es el término de error.

Posteriormente con el fin de examinar la relación de largo plazo entre las variables del modelo econométrico, planteamos un modelo de vectores auto regresivo (VAR) y 
se verificó la existencia de vectores de cointegración. En este modelo, todas las variables son endógenas y cada variable está en función de sus propios rezagos y los rezagos de las otras variables de la función. La longitud del rezago fue determinada con el criterio de información de Akaike
(1974). Asimismo, se considera el orden de integración de las variables mediante el test de Dickey y Fuller aumentado (1979), con el cual se determinó que todas las variables tienen un orden de integración I (0). El modelo VAR a estimar es el siguiente:

$$
\begin{aligned}
& \Delta P I B_{t}=\delta_{0}+\delta_{1} \sum_{t=1}^{a} \Delta P I B_{t-i}+\delta_{2} \sum_{t=1}^{a} \Delta E S_{t-i}+\delta_{3} \sum_{t=1}^{a} \delta E N S_{t-i}+u_{t 1} \\
& \Delta E S_{t}=\delta_{4}+\delta_{5} \sum_{t=1}^{a} \Delta E S_{t-i}+\delta_{6} \sum_{t=1}^{a} \Delta P I B_{t-i}+\delta_{7} \sum_{t=1}^{a} \Delta E N S_{t-i}+u_{t 2} \\
& \Delta E N S_{t}=\delta_{8}+\delta_{9} \sum_{t=1}^{a} \Delta E N S_{t-i}+\delta_{10} \sum_{t=1}^{a} \Delta P I B+\delta_{11} \sum_{t=1}^{a} \Delta E S_{t-i}+u_{t 3}
\end{aligned}
$$

Donde $\Delta$ es el operador de primeras diferencias. La longitud del rezago se define con el criterio de información de Akaike (1974). En la tercera etapa, una vez que la existencia de cointegración es verificada entre las variables del modelo, se obtiene el término de error de equilibrio $\delta_{i}$. Como sugiere Azlina y Mustapha (2012), este vector se puede utilizar para estimar un modelo de corrección de error (ECM) para determinar la existencia de equilibrio de corto plazo (Engle y Granger, 1987) entre las tres variables y la variable dummy de la estabilidad. La significación estadística del parámetro asociado con el error de equilibrio incorporado en el $E C T_{t-1}$ indica el mecanismo de corrección. El modelo ECM planteado esta expresado en la siguiente ecuación:

$$
\begin{gathered}
\Delta P I B_{t}=\alpha_{0}+\alpha_{1} \sum_{t=1}^{a} \alpha P I B_{t-i}+\alpha_{2} \sum_{t=1}^{a} \Delta E S_{t-i}+\alpha_{3} \sum_{t=1}^{a} \alpha E N S_{t-i}+\alpha_{4} E_{t-1}+\gamma_{t 1} \\
\Delta E S_{t}=\alpha_{5}+\alpha_{6} \sum_{t=1}^{a} \Delta E S_{t-i}+\alpha_{7} \sum_{t=1}^{a} \Delta P I B_{t-i}+\alpha_{8} \sum_{t=1}^{a} \Delta E N S_{t-i}+\alpha_{9} E_{t-1}+\gamma_{t 2} \\
\left.\Delta E N S_{t}=\alpha_{[} 10\right]+\alpha_{11} \sum_{t=1}^{a} \Delta E N S_{t-i}+\alpha_{12} \sum_{t=1}^{a} \Delta P I B+\alpha_{13} \sum_{t=1}^{a} \Delta E S_{t-i}+\alpha_{14} E_{t-1}+\gamma_{t 3}
\end{gathered}
$$

El test de cointegración de Johansen fue aplicado a las ecuaciones 4 y el test de corrección de error aplicado a las ecuaciones 5 incluye la variable dummy. Finalmente, a partir de las ecuaciones 4, se puede aplicar el test de causalidad de Granger (1979) para determinar la dirección de causalidad entre las tres variables.

Tabla 2 Resumen de estudio sobre el crecimiento económico y la energía sustentable y no sustentable

\begin{tabular}{cccccr}
\hline $\mathbf{N}^{\circ}$ & Autor y año & Países & Metodología & Variables & Resultados \\
\hline 1 & $\begin{array}{c}\text { Taghvaee, Mavuka y } \\
\text { Shirazi (2016) }\end{array}$ & Irán & Causalidad de Granger; VAR; VEC & CE; ER ENR & CE $\leftrightarrow$ CO \\
& Wang, Li, Fang y Zhou & China & Causalidad de Granger; VAR & CE;E; ECO2 & ECO2 $\rightarrow E$ \\
2 & (2015) & Canadá & Causalidad de Granger; VAR; VEC & CE; E & CE $\leftrightarrow$ E \\
3 & Ghaliy El-Sakka. (2004) & Malasia & Causalidad de Granger; VAR & CE $\rightarrow$ E \\
4 & Abd y Aziz (2011) & Nepal & Causalidad de Granger; VAR; VEC & CE; E; ECO2 & CE $\rightarrow$ E;ECO2 \\
5 & Bastola, Sapkota (2014), & Calim, Hassan, Shafiei, & Países de la OCDE & Causalidad de Granger; VAR; VEC & CE; ENR
\end{tabular}

Nota: $\rightarrow$,representa la causalidad unidireccional, $\leftrightarrow$ la causalidad bidireccional y $\neq$ no causalidad de Granger. Las abreviaturas se definen de la siguiente manera: $\mathrm{CE}=$ crecimiento económico, $\mathrm{ER}$ energía renovable, $\mathrm{ENR}=$ Energía no renovable $\mathrm{E}=$ consumo de energía, $\mathrm{ECO}=\mathrm{Emisiones} \mathrm{de}$ $\mathrm{CO} 2$. VAR $=$ vector autorregresivo, $\mathrm{VEC}=$ modelos de corrección de errores. 


\section{Discusión de resultados}

Discutiremos nuestros resultados con evidencia empírica que proponen la misma relación. Para poder comprobar la estacionalidad de las series aplicamos el test de Dickey y Fuller Aumentado, donde encontramos que efectivamente las variables son estacionarias, ya que todos los valores calculados de las tres variables en cuestión superan los valores críticos, lo que implica que tienen un orden de integración de orden I(0) como lo muestra la Tabla 3. Los resultados obtenidos mediante el test de Dickey y Fuller Aumentado (1979) son verificados mediante el test de Phillips y Perron
(1988), con tendencia e intercepto, respectivamente. En la práctica, los resultados son consistentes con ambos test que son los más utilizados de raíz unitaria. Este procedimiento es necesario para la estimación de econometría de series de tiempo para evitar resultados espúreos. Nuestros resultados son contrarios a los encontrados por Erdal, Erdal y Esengün (2008) en su estudio para el caso de Turquía en donde las variables tienen un orden de integración I(1), y contrarios a los encontrados por Fang y Chang (2016) y Mehrara (2017) en sus estudios para los países de Asia del pacífico y para los países exportadores de petróleo respectivamente.

Tabla 3 Prueba de Dickey y Fuller aumentada y de Phillips Perron

\begin{tabular}{|c|c|c|c|c|c|c|c|c|}
\hline & \multirow{3}{*}{$\begin{array}{l}\text { Valor } \\
\text { calculado }\end{array}$} & \multirow{2}{*}{\multicolumn{3}{|c|}{$\begin{array}{c}\text { Dickey y Fuller aumentado } \\
\text { Valor crítico }\end{array}$}} & \multirow{3}{*}{$\begin{array}{l}\text { Valor } \\
\text { calculado }\end{array}$} & \multirow{2}{*}{\multicolumn{3}{|c|}{$\begin{array}{c}\text { Phillips Perron } \\
\text { Valor crítico }\end{array}$}} \\
\hline & & & & & & & & \\
\hline & & $1 \%$ & $5 \%$ & $10 \%$ & & $1 \%$ & $5 \%$ & $10 \%$ \\
\hline$P I B p c_{t}$ & 4.31 & 3.63 & 2.95 & 2.61 & 4.33 & 3.63 & 2.95 & 2.61 \\
\hline$E S_{t}$ & 4.57 & 3.63 & 2.95 & 2.61 & 4.62 & 3.63 & 2.95 & 2.61 \\
\hline$E N S_{t}$ & 7.48 & 3.63 & 2.95 & 2.61 & 7.42 & 3.64 & 2.95 & 2.61 \\
\hline
\end{tabular}

Luego de haber verificado que las series si son estacionarias en niveles, estimamos la ecuación (2) y aplicamos un test de cointegración de Johansen (1988) para verificar la relación de largo plazo entre cada una de las variables. La Tabla 4 muestra los resultados del modelo VAR de la Energía sustentable y la energía no sustentable. El test de cointegración señala la existencia de un vector de cointegración entre el PIB, energía sustentable y energía no sustentable, por lo tanto, si existe equilibrio a largo plazo, estos resultados son coincidentes con Erdal, Erdal y Esengün (2008) quienes encontraron que el PIB y el consumo de energía no renovable están cointegración en el largo plazo en caso de Turquía y contrarios a los resultados encontrados por Dergiades et al. (2013) en su estudio para Grecia y contrarios a los resultados de Belke, Dobnik y Dreger (2011) en su estudio para 25 países de la OCDE. Nuestros allazgos también concuerdan con la invesyigación de Salim et al. (2014) en su estudio para los países de la OCDE.

Tabla 4 Resultados del test de cointegración de Johansen

\begin{tabular}{|c|c|c|c|c|c|}
\hline \multicolumn{6}{|c|}{ PIB/Energía sustentable } \\
\hline $\begin{array}{l}\text { Rango } \\
\text { máximo }\end{array}$ & Parámetros & $\mathrm{LL}$ & Valor propio & $\begin{array}{l}\text { Estadísticas de } \\
\text { rastreo }\end{array}$ & $5 \%$ valor crítico \\
\hline 0 & 12 & 203.424 & - & 35.24 & 29.68 \\
\hline 1 & 17 & 192.339 & 0.418 & $13.07^{*}$ & 15.41 \\
\hline 2 & 20 & 186.283 & 0.256 & 0.96 & 3.76 \\
\hline 3 & 21 & 185.805 & 0.023 & & \\
\hline \multicolumn{6}{|c|}{ PIB/Energía no sustentable } \\
\hline $\begin{array}{l}\text { Rango } \\
\text { máximo }\end{array}$ & Parámetros & LL & Valor propio & $\begin{array}{l}\text { Estadísticas de } \\
\text { rastreo }\end{array}$ & $5 \%$ valor crítico \\
\hline 0 & 12 & 219.769 & - & 42.39 & 29.68 \\
\hline 1 & 17 & 204.872 & 0.517 & $12.60 *$ & 15.41 \\
\hline 2 & 20 & 198.937 & 0.251 & 0.73 & 3.76 \\
\hline 3 & 21 & 198.571 & 0.017 & & \\
\hline
\end{tabular}


Después de haber analizado el modelo VAR, en donde los resultados muestran la existencia de relación a largo plazo entre las variables usadas en el modelo, también hemos planteado un modelo de corrección de error VEC (Engle y Granger, 1987), donde podemos observar la Tabla 5 muestra los resultados de la estimación del modelo de corrección de error VEC, donde podemos observar que si existe relación causal a corto plazo ya que el rezago del error resulta significativo, resultados concordantes con los resultados obtenidos por Tang et al. (2016) en su estudio para Vietnam en donde encuentra que el crecimiento económico y el consumo de energía están cointegrados en el corto plazo, Ghali et al. (2004) también encuentra vectores de cointegración en el corto plazo en su estudio para Canadá y también concordantes con los resultados de Salim et al. (2014) quién también encuentra vectores de cointegración en el corto plazo.

Tabla 5 Resultados de la estimación del modelo de corrección de error VEC

\begin{tabular}{|c|c|c|c|c|c|c|}
\hline \multirow{2}{*}{$\begin{array}{l}\text { Beta } \\
\text { cel }\end{array}$} & Coef. & Std. Err & $\mathrm{Z}$ & $P>|z|$ & \multicolumn{2}{|c|}{ [95\% Conf. Interval.] } \\
\hline & 1 & & & & & \\
\hline$E S_{t}$ & 1.756 .243 & $1.49 \mathrm{e}-08$ & $1.2 \mathrm{e}+08$ & 0.000 & 1.756 .243 & 1.756 .243 \\
\hline$E N S_{t}$ & -263.376 & $1.20 \mathrm{e}-08$ & $-2.2 \mathrm{e}+08$ & 0.000 & -263.376 & -263.376 \\
\hline Dic & -4.746 .907 & $1.58 \mathrm{e}-07$ & $-3.0 e+07$ & 0.000 & -4.746 .907 & -4.746 .907 \\
\hline $\mathrm{Cel}$ & -1 & $4.35 \mathrm{e}-09$ & $-2.3 \mathrm{e}+08$ & 0.000 & -1 & -1 \\
\hline cons & -3.933 .805 & & & & & \\
\hline
\end{tabular}

Finalmente analizamos el test de causalidad de Granger (1969), el cual señala que existe causalidad entre las variables, energía sustentable causa al crecimiento económico $(E S \rightarrow P I B p c)$, siendo esta una relación unidireccional. Esta relación se presenta en la Tabla 6. Nuestros resultados son parecidos a los obtenidos por Ghali (2004) quién encuentra causalidad bidireccional entre el crecimiento económico y el consumo de energía. Bowden y Payne (2009) también encuentra causalidad de Granger bidireccional entre el consumo de energía primaria comercial, residencial, primaria industrial y el PIB real, en un estudio para Estados Unidos. Nuestros resultados coindicen de mejor manera con los resultados encontrados por Lee (2005) quién demuestra causalidad unidireccional desde el consumo de energía hacia el crecimiento económico en su estudio para países en desarrollo y también con los estudios de Salim et al. (2014) quién encuentra causalidad unidireccional en el mismo sentido, pero es bidireccional cuando la relación es en el corto plazo.

Tabla 6 Resultados de las pruebas de causalidad de Granger

\begin{tabular}{lcccc} 
Equation & Excluded & chi2 & Df & Prob $>$ chi2 \\
\hline Energía sustentable & PIBpc & 7.8004 & 2 & 0.020
\end{tabular}

\section{Conclusiones e implicaciones}

La presente investigación demuestra que las energías sustentables contribuyen positivamente al crecimiento económico del Ecuador, tomado en cuenta que este tipo de energías además de no contaminar el planeta donde vivimos, tiene costes que son sumamente bajos en comparación a los combustibles fósiles. Hoy en día el crecimiento de energía limpia ha ido en aumento, recibiendo un importante respaldo de las grandes potencias mundiales, que son los que más invierten en este tipo de energía mejorando sustancialmente su crecimiento económico. En Ecuador se observan grandes avances gracias al trabajo en el cambio de la matriz energética que ha contribuido que el país hoy en día consuma un 51,78\% de energía limpia. los resultados que obtuvimos muestran efectivamente que el consumo de energía sustentables y no sustentable tiene una relación positiva a largo y a corto plazo con el crecimiento económico. Además, a través de las pruebas de causalidad de Granger (1969), encontramos la existencia de causalidad unidireccional, es decir, la energía sustentable causa al crecimiento económico. muchos de los autores sugieren que se debería seguir invirtiendo en energías limpias y dejar de lado las energías que causan contaminación. Futuras investigaciones podrían profundizar en estrategias para que el país pueda reducir su dependencia de los combustibles fósiles y se incline por energías sustentables mediante la oferta, siguiendo con mayor cantidad de inversión para lograr alcanzarlas metas trazadas por el gobierno, y por el lado de la demanda ofrecer incentivos o subsidios de manera que se pueda lograr reemplazar la energía contaminante con una energía amigable con el medio ambiente en el que vivimos.

\section{Referencias Bibliográficas}

Al-Mulali, U., Fereidouni, H. G., y Lee, J. Y. (2014). Electricity consumption from renewable and nonrenewable sources and economic growth: Evidence from Latin American countries. Renewable and Sustainable Energy Reviews, 30, 290-298. doi: 10.1016/ j.rser.2013.10.006

Aziz, A. A. (2011). On the Causal Links between Energy Consumption and Economic Growth in Malaysia. International Review of Business Research Papers, 7(6), 180-189.

Bastola, U., y Sapkota, P. (2015). Relationships among energy consumption, pollution emission, and economic growth in Nepal. Energy, 80, 254-262. doi: 10.1016/j.energy.2014.11.068

Belke, A., Dobnik, F., y Dreger, C. (2011). Energy consumption and economic growth: New insights into the cointegration relationship. Energy Economics, 33(5), 782-789. doi: 10.1016/j.eneco.2011.02.005

Ben, M., Ben, S., y Ozturk, I. (2017). Testing environmental Kuznets curve hypothesis: The role of renewable and non-renewable energy consumption and trade in OECD countries. , 60(2016), 824-831.

Bowden, N., y Payne, J. E. (2009). The causal relationship between U.S. energy consumption and real output: A disaggregated analysis. Journal of Policy Mode- 
ling, 31(2), 180-188. doi: 10.1016/j.jpolmod.2008 .09 .001

Chiou-wei, S. Z., Chen, C.-f., y Zhu, Z. (2012). Economic growth and energy consumption revisited - Evidence from linear and nonlinear Granger causality. , 30(2008), 3063-3076. doi: 10.1016/j.eneco.2008.02 .002

Dergiades, T., Martinopoulos, G., y Tsoulfidis, L. (2013). Energy consumption and economic growth: Parametric and non-parametric causality testing for the case of Greece. Energy Economics, 36, 686-697. doi: 10.1016/j.eneco.2012.11.017

Esseghir, A., y Haouaoui Khouni, L. (2014). Economic growth, energy consumption and sustainable development: The case of the Union for the Mediterranean countries. Energy, 71, 218-225. doi: 10.1016/ j.energy.2014.04.050

Fang, Z., y Chang, Y. (2016). Energy, human capital and economic growth in Asia Pacific countries Evidence from a panel cointegration and causality analysis. Energy Economics, 56, 177-184. doi: 10.1016/j.eneco.2016.03.020

Ghali, K. H. (2004). Energy use and output growth in Canada : a multivariate cointegration analysis. , 26, 225-238. doi: 10.1016/S0140-9883

Hondroyiannis, G., Lolos, S., y Papapetrou, E. (2002). Energy consumption and economic growth: Assessing the evidence from Greece. Energy Economics, 24(4), 319-336. doi: 10.1016/S0140-9883(02)00006 $-3$

Long, X., Naminse, E. Y., Du, J., y Zhuang, J. (2015). Nonrenewable energy, renewable energy, carbon dioxide emissions and economic growth in China from 1952 to 2012. Renewable and Sustainable Energy Reviews, 52, 680-688. doi: 10.1016/j.rser.2015.07.176

Maji, I. K. (2015). Does clean energy contributes to economic growth? Evidence from Nigeria. Energy Reports, 1, 145-150. doi: 10.1016/j.egyr.2015.06.001

Mehrara, M. (2007). Energy consumption and economic growth: The case of oil exporting countries. Energy Policy, 35(5), 2939-2945. doi: 10.1016/j.enpol.2006 .10 .018

Ohler, A., y Fetters, I. (2014). The causal relationship between renewable electricity generation and GDP growth: A study of energy sources. Energy Economics, 43, 125-139. doi: 10.1016/j.eneco.2014.02 .009

Pablo-Romero, M. D. P., y De Jesús, J. (2016). Economic growth and energy consumption: The EnergyEnvironmental Kuznets Curve for Latin America and the Caribbean. Renewable and Sustainable Energy Reviews, 60, 1343-1350. doi: 10.1016/j.rser.2016.03
.029

REN21. (2015). Reporte de la situación mundial de las energías renovables en 2015. Renewables 2015 Global Status Report., 32.

Saboori, B., y Sulaiman, J. (2013). CO2emissions, energy consumption and economic growth in association of Southeast Asian Nations (ASEAN) countries: Acointegration approach. Energy, 55, 813-822. doi: 10.1016/j.energy.2013.04.038

Salahuddin, M., y Alam, K. (2015). Internet usage, electricity consumption and economic growth in Australia: A time series evidence. Telematics and Informatics, 32(4), 862-878. doi: 10.1016/j.tele.2015.04.011

Salim, R. A., Hassan, K., y Shafiei, S. (2014). Renewable and non-renewable energy consumption and economic activities: Further evidence from OECD countries. Energy Economics, 44, 350-360. doi: 10.1016/j.eneco.2014.05.001

Taghvaee, V. M., Mavuka, C., y Shirazi, J. K. (2016). Economic growth and energy consumption in Iran : an ARDL approach including renewable and nonrenewable energies. Environment, Development and Sustainability. doi: 10.1007/s10668-016-9862-Z

Wang, S., Li, Q., Fang, C., y Zhou, C. (2016). Science of the Total Environment The relationship between economic growth, energy consumption, and CO 2 emissions : Empirical evidence from China. Science of the Total Environment, The, 542, 360-371. doi: 10.1016/j.scitotenv.2015.10.027

Zaman, K., Abdullah, A. B., Khan, A., Nasir, M. R. B. M., Hamzah, T. A. A. T., y Hussain, S. (2016). Dynamic linkages among energy consumption, environment, health and wealth in BRICS countries: Green growth key to sustainable development. Renewable and Sustainable Energy Reviews, 56, 1263-1271. doi: 10.1016/j.rser.2015.12.010

Recibido: 30 de junio de 2018

Aceptado: 3 de septiembre de 2018 
